\title{
Studying fitness cost of Plasmodium falciparum infection in malaria vectors: validation of an appropriate negative control
}

Ibrahim Sangare ${ }^{1,2}$, Yannis Michalakis ${ }^{2}$, Bienvenue Yameogo ${ }^{1}$, Roch Dabire ${ }^{1}$, Isabelle Morlais ${ }^{2,3}$ and Anna Cohuet ${ }^{1,2^{*}}$

\begin{abstract}
Background: The question whether Plasmodium falciparum infection affects the fitness of mosquito vectors remains open. A hurdle for resolving this question is the lack of appropriate control, non-infected mosquitoes that can be compared to the infected ones. It was shown recently that heating $P$. falciparum gametocyte-infected blood before feeding by malaria vectors inhibits the infection. Therefore, the same source of gametocyte-infected blood could be divided in two parts, one heated, serving as the control, the other unheated, allowing the comparison of infected and uninfected mosquitoes which fed on exactly the same blood otherwise. However, before using this method for characterizing the cost of infection to mosquitoes, it is necessary to establish whether feeding on previously heated blood affects the survival and fecundity of mosquito females.

Methods: Anopheles gambiae M molecular form females were exposed to heated versus non-heated, parasite-free human blood to mimic blood meal on non-infectious versus infectious gametocyte-containing blood. Life history traits of mosquito females fed on blood that was heat-treated or not were then compared.

Results: The results reveal that heat treatment of the blood did not affect the survival and fecundity of mosquito females. Consistently, blood heat treatment did not affect the quantity of blood ingested.

Conclusions: The study indicates that heat inactivation of gametocyte-infected blood will only inhibit mosquito infection and that this method is suitable for quantifying the fitness cost incurred by mosquitoes upon infection by P. falciparum.
\end{abstract}

Keywords: Plasmodium falciparum, Vectors, Infection cost, Negative control, Fitness

\section{Background}

Mosquito fitness is a crucial factor of vectorial capacity and malaria transmission [1]. However, the question whether Plasmodium parasites affect the fitness of mosquito vectors they infect remains open. Ten years ago, a review pointed out conflicting results in previous studies according to the design of experiments, environmental conditions, and the parasite-mosquito species used [2]. This underlined the need to develop experiments using natural vector-parasite species combinations in controlled environmental conditions. In the last decade, additional

\footnotetext{
* Correspondence: anna.cohuet@ird.fr

${ }^{1}$ Institut de Recherche en Sciences de la Santé-Direction Régionale de

I'Ouest, Bobo Dioulasso, Burkina Faso

${ }^{2}$ Institut de Recherche pour le Développement, Unité MIVEGEC (IRD 224-

CNRS 5290-UM1-UM2), BP 64501, Montpellier Cedex 5 34394, France

Full list of author information is available at the end of the article
}

studies aimed at deciphering the cost of infection in malaria vectors by using different species and methods and produced again contrasted results [3-7]. Some studies showed a shorter lifespan in infected mosquitoes or no detected effect of infection depending on the parasite load and/or environmental conditions [3,5-7]. Recently, another study observed a longer lifespan in infected mosquitoes associated with a lower fecundity [4]. Concerning the most important vectorial system for human malaria: Anopheles gambiae/Plasmodium falciparum, some studies suggested an infection cost in this combination of species $[7,8]$, others did not find it $[9,10]$. Further investigations are therefore needed to determine how infection affects the mosquito vector in this epidemiologically relevant system.

\section{Biomed Central}


Measuring how parasite infection affects fitness traits requires the comparison of infected and non-infected mosquitoes, either collected from the field or produced in laboratory conditions. Comparison of field-collected infected and non-infected mosquitoes is limited by the difficulties to obtain a large number of infected mosquitoes [7], and by the lack of a reliable method for ageing the fieldcollected mosquitoes [8]. In the laboratory, obtaining a large number of Plasmodium-infected mosquitoes is facilitated by exposing insectary-reared Anopheles to gametocytes through membrane feeding (gametocyte-infected blood from naturally infected patients in direct membrane feeding assays, DMFA, or gametocyte-containing parasite culture in standard membrane feeding assays, SMFA) [11]. Different methods were used so far for producing noninfected mosquitoes to be compared to the ones exposed to Plasmodium gametocytes. In DMFA, in parallel to the experimental infection of mosquitoes, other females were exposed to parasite-free blood from another volunteer $[10,12]$; in SMFA the control mosquitoes were fed on a parasite culture not producing gametocytes [13]. Also exposing the mosquitoes that ingested infectious gametocytes to high temperatures just after the blood feeding can limit the infection success and produce non-infected mosquitoes [13]. All these methods however, incur the problem of confounding factors: when using the blood of uninfected hosts, one cannot rule out that host effects are responsible for the potential differences in mosquito life-history traits [14]; also, parasite strain-specific effects, or temperature effects $[15,16]$ may be confounded with the effect of infection. This underlines a need for a proper control for evaluating the cost of infection.

It was shown recently that heating $P$. falciparum gametocyte-infected blood before feeding by malaria vectors impedes the infection by killing or inhibiting the infectivity of gametocytes $[17,18]$. This potentially allows the comparison of infected to non-infected mosquitoes that fed on the same blood at the same time. However, before using this method for characterizing the cost of infection to mosquitoes, it is necessary to establish whether feeding on previously heated blood affects the survival and fecundity of mosquito females. Therefore, life history traits of female mosquitoes exposed to noninfected blood that was subjected or not to a heat treatment, were compared. This allowed validating the method of using heat inactivation of $P$. falciparum gametocytes for comparison of fitness traits between infected and non-infected An. gambiae.

\section{Methods}

\section{Mosquitoes}

A mosquito colony of An. gambiae M molecular form was used. The colony was established in 2008 from wild-caught gravid females collected in Kou Valley $(30 \mathrm{~km}$ north from
Bobo Dioulasso), Burkina Faso [19]. The females laid eggs individually before their species was determined by PCRRFLP [20] and M form egg batches were pooled. The colony was maintained in the insectary under standard conditions (12 h day/night cycle, $28+/-2^{\circ} \mathrm{C}, 80+/-5 \%$ humidity).

The first blood meal of An. gambiae females is potentially used to compensate for nutritional deficiencies carried over from larval life and induce complete egg development and laying only in bigger females, whereas almost all females are able to fully develop eggs at subsequent blood meals [21-23]. The second blood meal is therefore more representative of female fecundity along its lifespan and egg production was observed after the second blood meal. Before measures of life history traits, females were fed on rabbit blood three days after adult emergence and on human blood the fifth or sixth day of adult life. Sugar was removed $36 \mathrm{~h}$ prior to blood feeding. For mosquito feeding on human blood, venous blood was collected from five volunteers, non-infected by P. falciparum, who were enrolled after written informed consent. Ethical approval was obtained from the relevant institutional Ethics Committee.

\section{Blood feeding}

Non-infected human blood was treated in the same manner as for experimental infections of malaria vectors by DMFA using natural isolates of $P$. falciparum and using gametocyte heat inactivation in parallel $[17,18]$. The blood was first centrifuged at $2,000 \mathrm{rpm}$ at $37^{\circ} \mathrm{C}$ for three min, and the serum was replaced by the same volume of European $A B$ serum. In experimental infections, this step limits the effect of human transmission blocking immunity [24]. To mimic gametocyte heat inactivation, half of the reconstituted blood was placed in a thermo-mixer and heated at $43^{\circ} \mathrm{C}$ for $15 \mathrm{~min}$ and $900 \mathrm{rpm}$ while the remaining blood was maintained at $37^{\circ} \mathrm{C}$. Five hundred $\mu \mathrm{l}$ of blood (heated or not) were distributed in membrane feeders maintained at $37^{\circ} \mathrm{C}$ by water jackets. At least two different feeders were used for each group (blood donor and heat treatment) in order to limit potential feeder effects. Cups containing 50 mosquito females were placed under the feeders to allow blood feeding through Parafilm membranes for $30 \mathrm{~min}$. Fed females were sorted and placed in individual $30 \mathrm{ml}$ plastic tubes for subsequent measures of life history traits. Because of logistic limitations, the blood feeding with the different blood donors had to be carried out at different days and using different mosquito batches from the same colony and therefore constituted different experimental blocks. In the statistical analyses the effect "blood donor" could thus be due to either differences in the "quality" of the blood of the various donors, intrinsic differences of the different mosquito batches, date effects, or a combination of the above. 
Table 1 Statistical analysis of effects on Anopheles gambiae females survival

\begin{tabular}{llll}
\hline Source & DF & $\boldsymbol{X}^{\mathbf{2}}$ & $\boldsymbol{P}$ value \\
\hline Heat treatment & 1 & 1.441 & 0.2300 \\
\hline Blood donor & 4 & 95.705 & $<.0001$ \\
\hline Heat treatment* Blood donor & 4 & 3.114 & 0.5390 \\
\hline Wing length & 1 & 8.087 & 0.0045 \\
\hline Haematin & 1 & 10.240 & 0.0014
\end{tabular}

Analysis of the effect of blood heat treatment, blood donor and their interaction on female mosquito survival after the blood meal using the Cox Proportional Hazards model.

\section{Measure of life history traits}

From the day of membrane blood feeding on heated versus non-heated blood and until death of all mosquitoes, females were observed every eight hours. Dead females were immediately processed: ovaries were dissected for egg counting and wings were mounted on slides to measure their length. If both wings of a female were intact one wing was randomly chosen to be measured. When one wing was damaged, the undamaged wing was measured. Wing size was measured from the alula to the wing tip, excluding scales as previously described [25] by using the software ImageJ [26].

The amount of haematin, a by-product of the decomposition of haemoglobin, was measured for each female [27] to obtain a relative measure of blood meal size. For all mosquitoes that died at least three days after blood feeding and therefore completed digestion and excreted all the haematin, $1 \mathrm{ml}$ of $1 \% \mathrm{LiCO}_{3}$ was distributed in individual tubes to elute faeces. The absorbance of the resulting solution was read at $387 \mathrm{~nm}$, using $\mathrm{LiCO}_{3}$ solution as a blank, and compared with a standard curve made with porcine serum haematin (Sigma-Aldrich).

\section{Statistical analyses}

To investigate whether the blood heating treatment affects mosquito survival, Kaplan-Meier survival estimates were calculated and the effects of heat treatment, blood donor, and their interaction, were tested using Cox's Proportional Hazards model. Wing length and the quantity of excreted haematin were used as co-variables.

Preliminary analysis of the results on fecundity revealed that a variable proportion of females did not develop any eggs, thus yielding distributions with a spike at zero and a more or less normal distribution for larger values of the number of eggs produced. The analysis of fecundity results were thus split in two parts. First, nominal logistic regressions were used to test whether heat treatment affects the probability that a female may develop at least one egg. Because the size of an individual and its blood meal size may affect female fecundity wing length and quantity of haematin excreted were included as co-variables in the model. Second, the effect of blood treatment on the number of eggs developed per female, exclusively on females that developed at least one egg, was tested with an analysis of variance including wing length and quantity of haematin excreted as co-variables.

In order to investigate whether heating the blood may influence the quantity ingested by a female, the potential effect of the heat treatment on the blood meal size was tested with an analysis of variance with blood donor as a co-variable.

In the analyses, the blood-donor effect is considered as fixed. The choice of this option was done because (i) fixed effects models are easier to present than mixed effects models; (ii) when declaring the blood-donor effect and its interaction with the heat treatment as random effects, similar conclusions were always reached; (iii) the small number of blood donors (five) legitimately questions whether they can be considered as a representative sample of the blood donor population.
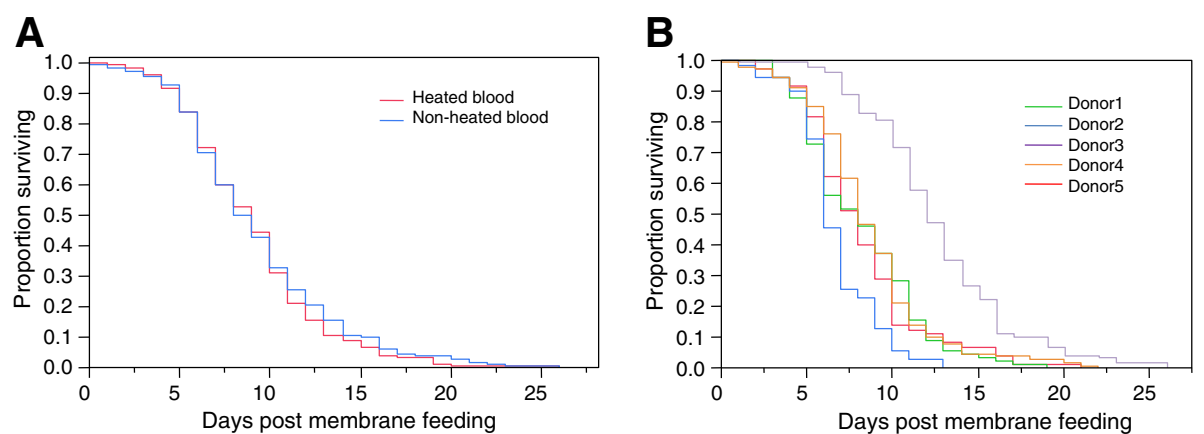

Figure 1 Survival curves. Kaplan-Meier estimates of survival of female mosquitoes after a blood meal as a function of whether the blood had been previously heated (in red) or not (in blue) (A) or as a function of blood donor (B). In A, survival curves between females fed on heated versus non-heated blood are not significantly different according to the Cox Proportional Hazards tests ( $P=0.46)$. In $B$, survival curves between females fed on blood from different blood donors are significantly different according to the Cox Proportional Hazards tests (P <0.001). 
Table 2 Statistical analysis of effects on development of at least one egg in Anopheles gambiae

\begin{tabular}{llll}
\hline Source & DF & $\boldsymbol{X}^{\mathbf{2}}$ & $\boldsymbol{P}$ value \\
\hline Heat treatment & 1 & 0.304 & 0.5816 \\
\hline Blood donor & 4 & 5.611 & 0.2302 \\
\hline Heat treatment *Blood donor & 4 & 1.829 & 0.7672 \\
\hline Wing length & 1 & 1.811 & 0.1784 \\
\hline Haematin & 1 & 20.151 & $<.0001$
\end{tabular}

Nominal logistic regression on whether a female laid any eggs as a function of blood treatment, blood donor, their interaction, female wing length and quantity of haematin excreted.

\section{Results}

\section{Survival}

A total of 485 An. gambiae females were included in the analysis, ranging from 70 to 131 per blood donor. The Cox Proportional Hazards model revealed that the blood heating treatment, whether alone or in interaction with the blood donor, did not affect mosquito survival (Table 1, Figure 1A). In contrast, a significant difference in survival among females that fed on blood from different donors was evidenced (Table 1, Figure 1B). Similar conclusions were obtained if instead of carrying out the analysis on survival after the membrane blood meal the total adult lifespan since emergence was used (results not shown). Longevity was highly different between mosquito batches fed on different blood donors, with median day of death post membrane feeding ranging from six (blood donor 2) to 12 (blood donor 3), corresponding to 11 and 17 days post-emergence respectively. Longevity was also significantly affected by wing length and the quantity of haematin excreted (Table 1). The estimates indicated that the former relation is negative and the latter positive (not shown). This suggests a longer lifespan for the females that ingested more blood, while larger females died faster.

\section{Fecundity}

The proportion of females that did not develop any egg varied significantly among blood donors and was negatively influenced by blood meal size: females that feed more blood were more prone to develop at least one egg. In contrast, the proportion of females that did not develop any egg was not affected by the blood treatment

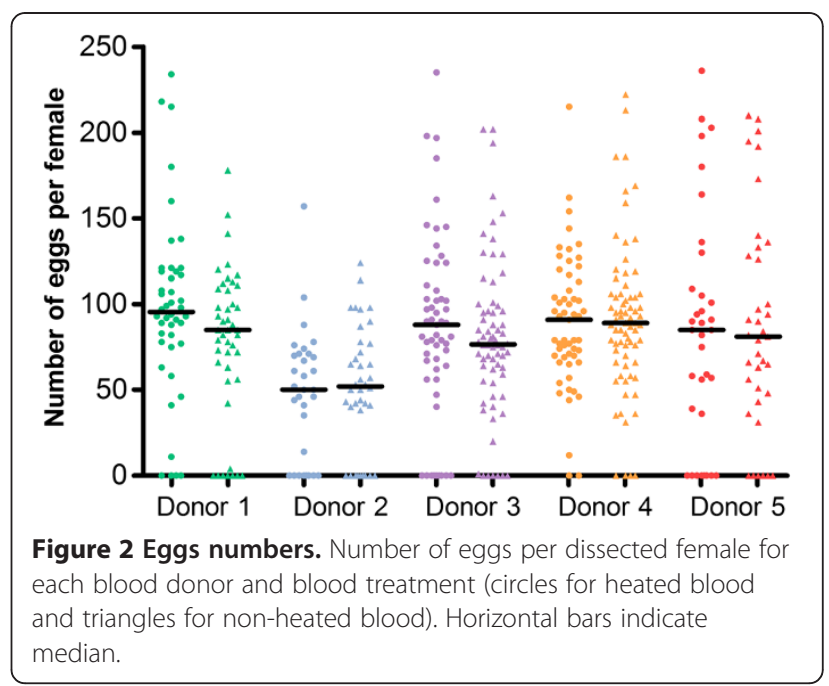

or female wing length (Table 2). For females that developed at least one egg, blood treatment did not affect the number of eggs developed by each female. There was significant variation among donors, the average number of eggs per female across patients ranging from 66 to 107 (Table 3, Figure 2). The number of eggs was also significantly larger in bigger females and females that ingested larger blood meals (Table 3).

\section{Blood meal size}

The analysis did not detect any effect of blood heat treatment on the quantity of blood ingested by female mosquitoes. However, the donor effect was significant (Table 4), suggesting that the blood meal size taken by the mosquitoes differs between blood donor.

\section{Discussion}

In the present study, it was investigated whether a heat treatment of human blood that mimics heat inactivation of $P$. falciparum gametocytes affects the fitness of the vector mosquitoes. Life history traits of An. gambiae females fed on parasite-free blood that was heat treated to that of females fed on not-treated blood were compared. The results revealed that heat treatment of the blood did not affect the survival and fecundity of mosquito females. Consistently, blood heat treatment did

Table 3 Statistical analysis of effects on number of eggs developed in Anopheles gambiae

\begin{tabular}{|c|c|c|c|c|}
\hline Source & DF & Sum of squares & F ratio & Prob $>F$ \\
\hline Heat treatment & 1 & 1921.585 & 1.3775 & 0.2414 \\
\hline Blood donor & 4 & 24304.107 & 4.3557 & 0.0019 \\
\hline Heat treatment *Blood donor & 4 & 8354.035 & 1.4972 & 0.2027 \\
\hline Wing length & 1 & 68160.616 & 48.8617 & $<.0001$ \\
\hline Haematin & 1 & 3889.278 & 2.7881 & 0.0959 \\
\hline
\end{tabular}

Analysis of variance of the number of eggs developed per female as a function of blood treatment, blood donor and their interaction, female wing length and haematin excreted (model $R^{2}=0.24$ ). 
Table 4 Statistical analysis of effects on blood meal size in Anopheles gambiae

\begin{tabular}{llllll}
\hline Source & DF & Sum of squares & F ratio & \\
\hline Heat treatment & 1 & 3.4092 & 0.0756 & Prob $>$ F \\
\hline Blood donor & 4 & 1165.2930 & 6.4596 & 0.7835 \\
\hline Heat treatment *Blood donor & 4 & 346.0512 & 1.9183 & $<.0001$ \\
\hline
\end{tabular}

Analysis of variance of the quantity of haematin excreted as a function of blood treatment and blood donor (model $\mathrm{R}^{2}=0.07$ ).

not affect the quantity of blood ingested. Then, it can be conjectured that heat inactivation of gametocyteinfected blood will only inhibit mosquito infection and that this method is suitable for quantifying the fitness cost incurred by mosquitoes upon an infection by $P$. falciparum.

Mosquito body size can affect both longevity and fecundity e.g. [21,28-30], either directly or indirectly through its potential effect on the quantity of ingested blood $[31,32]$. These variables were therefore included into the analysis. The results evidenced positive relations of both the body size, estimated by wing length, and the blood meal size, estimated from the quantity of haematin excreted, with the number of eggs developed. This confirms that bigger females and the ones that ingest more blood have higher fecundity, as previously observed in $A n$. gambiae and other mosquito species [33-35]. Blood meal size was also found positively related to longevity but bigger females were found to die faster. This result indicates that nutritive resources of the blood meal are used by the mosquito females for survival; however there is no clear explanation for why larger females died faster in this experiment. A trade-off between fecundity and longevity could explain this relation, but the data did not support this hypothesis.

The present study revealed large variations in fecundity and longevity of mosquitoes between replicates. The replicates differed by the batch of mosquitoes, taken from same mosquito colony, the day of experiment, and the blood donor. It is therefore not possible to conclude whether differences are due to blood characteristics or uncontrolled variations in insectary conditions that affect mosquito fitness. Further experiments will be needed to determine the effect of human blood variations on mosquito fitness, which could be a key parameter of mosquito preference for blood feeding and then malaria transmission [36,37].

\section{Conclusion}

This study aimed at validating an accurate method for producing control non-infected mosquitoes to be compared with $P$. falciparum-infected mosquitoes for measures of life history traits. The blood heat treatment at $43^{\circ} \mathrm{C}$ for $15 \mathrm{~min}$ and $900 \mathrm{rpm}$, which was previously demonstrated to effectively hinder $P$. falciparum infectivity, does not affect mosquito longevity and fecundity.
Therefore, this method can be used to feed simultaneously mosquitoes on gametocyte-containing blood versus heat-inactivated blood from the same donor. This procedure will enable accurate characterization of the cost of $P$. falciparum infection incurred by mosquitoes while avoiding potentially confounding factors from parasites or blood donors.

\section{Competing interests}

The authors declare that they have no competing interests.

\section{Authors' contributions}

IS, BY carried out the experiments, IS drafted the manuscript. YM participated in the design of the study and carried out the statistical analysis. RD and IM participated in the design of the study. AC conceived of the study, and participated in its design and coordination. All authors read and approved the final manuscript.

\section{Acknowledgements}

The research leading to these results has received funding from the French "Agence Nationale pour la Recherche" (ANR-06-MIME-001-01), from the European Community's Seventh Framework Programme (FP7/2007-2013) under grant agreements $N^{\circ} 242095$ and $N^{\circ} 223736$. This work was also supported by a scholarship from DSF-IRD to IS.

\section{Author details}

${ }^{1}$ Institut de Recherche en Sciences de la Santé-Direction Régionale de I'Ouest, Bobo Dioulasso, Burkina Faso. ${ }^{2}$ Institut de Recherche pour le Développement, Unité MIVEGEC (IRD 224-CNRS 5290-UM1-UM2), BP 64501, Montpellier Cedex 5 34394, France. ${ }^{3}$ Laboratoire de Recherche sur le Paludisme, Organisation de Coordination pour la lutte contre les Endémies en Afrique Centrale, Yaoundé BP 288, Cameroon.

Received: 20 September 2012 Accepted: 29 December 2012 Published: 2 January 2013

\section{References}

1. Smith DL, McKenzie FE: Statics and dynamics of malaria infection in Anopheles mosquitoes. Malar J 2004, 3:13.

2. Ferguson HM, Read AF: Why is the effect of malaria parasites on mosquito survival still unresolved? Trends Parasitol 2002, 18:256-261.

3. Lambrechts L, Chavatte JM, Snounou G, Koella JC: Environmental influence on the genetic basis of mosquito resistance to malaria parasites. Proc Biol Sci 2006, 273:1501-1506.

4. Vezilier J, Nicot A, Gandon S, Rivero A: Plasmodium infection decreases fecundity and increases survival of mosquitoes. Proc Biol Sci 2012, 279:4033-4041.

5. Ferguson HM, Mackinnon MJ, Chan BH, Read AF: Mosquito mortality and the evolution of malaria virulence. Evolution 2003, 57:2792-2804.

6. Dawes EJ, Churcher TS, Zhuang S, Sinden RE, Basanez MG: Anopheles mortality is both age- and Plasmodium-density dependent: implications for malaria transmission. Malar J 2009, 8:228.

7. Aboagye-Antwi F, Guindo A, Traore AS, Hurd H, Coulibaly M, Traore S, Tripet F: Hydric stress-dependent effects of Plasmodium falciparum infection on the survival of wild-caught Anopheles gambiae female mosquitoes. Malar J 2010, 9:243.

8. Hogg JC, Hurd H: The effects of natural Plasmodium falciparum infection on the fecundity and mortality of Anopheles gambiae s. I. in north east Tanzania. Parasitology 1997, 114(Pt 4):325-331. 
9. Chege GM, Beier JC: Effect of Plasmodium falciparum on the survival of naturally infected afrotropical Anopheles (Diptera: Culicidae). J Med Entomol 1990, 27:454-458.

10. Robert V, Verhave JP, Carnevale P: Plasmodium falciparum infection does not increase the precocious mortality rate of Anopheles gambiae. Trans $R$ Soc Trop Med Hyg 1990, 84:346-347.

11. Bousema T, Drakeley C: Epidemiology and infectivity of Plasmodium falciparum and Plasmodium vivax gametocytes in relation to malaria control and elimination. Clin Microbiol Rev 2011, 24:377-410.

12. Lavazec C, Boudin C, Lacroix R, Bonnet S, Diop A, Thiberge S, Boisson B, Tahar R, Bourgouin C: Carboxypeptidases B of Anopheles gambiae as targets for a Plasmodium falciparum transmission-blocking vaccine. Infect Immun 2007, 75:1635-1642.

13. Rodrigues J, Brayner FA, Alves LC, Dixit R, Barillas-Mury C: Science 2010, 329:1353-1355

14. Ferguson HM, Rivero A, Read AF: The influence of malaria parasite genetic diversity and anaemia on mosquito feeding and fecundity. Parasitology 2003, 127(Pt 1):9-19.

15. Blanford S, Read AF, Thomas MB: Thermal behaviour of Anopheles stephensi in response to infection with malaria and fungal entomopathogens. Malar J 2009, 8:72.

16. Paaijmans KP, Blanford S, Bell AS, Blanford JI, Read AF, Thomas MB: Influence of climate on malaria transmission depends on daily temperature variation. Proc Natl Acad Sci USA 2010, 107:15135-15139.

17. Mendes AM, Awono-Ambene PH, Nsango SE, Cohuet A, Fontenille D, Kafatos FC, Christophides GK, Morlais I, Vlachou D: Infection intensitydependent responses of Anopheles gambiae to the African malaria parasite Plasmodium falciparum. Infect Immun 2011, 79:4708-4715.

18. Mendes AM, Schlegelmilch T, Cohuet A, Awono-Ambene P, De lorio M, Fontenille D, Morlais I, Christophides GK, Kafatos FC, Vlachou D: Conserved mosquito/parasite interactions affect development of Plasmodium falciparum in Africa. PLoS Pathog 2008, 4:e1000069.

19. Harris C, Morlais I, Churcher TS, Awono-Ambene P, Gouagna LC, Dabire RK, Fontenille D, Cohuet A: Plasmodium falciparum produce lower infection intensities in local versus foreign Anopheles gambiae populations. PLOS One 2012, 7:e30849.

20. Fanello C, Santolamazza F, Della Torre A: Simultaneous identification of species and molecular forms of the Anopheles gambiae complex by PCRRFLP. Med Vet Entomol 2002, 16:461-464.

21. Takken W, Klowden MJ, Chambers GM: Effect of body size on host seeking and blood meal utilization in Anopheles gambiae sensu stricto (Diptera: Culicidae): the disadvantage of being small. J Med Entomol 1998, 35:639-645.

22. Charlwood JD, Pinto J, Sousa CA, Ferreira C, Petrarca V, Rosario Vdo E: A mate or a meal'-pre-gravid behaviour of female Anopheles gambiae from the islands of Sao Tome and Principe, West Africa. Malar J 2003, 2:9.

23. Mouline K, Mamai W, Agnew P, Tchonfienet M, Brengues C, Dabire R, Robert $V$, Simard F: Physiology and development of the $M$ and $S$ molecular forms of Anopheles gambiae in Burkina Faso (West Africa). Med Vet Entomol 2012, 26:447-454.

24. Boudin C, Diop A, Gaye A, Gadiaga L, Gouagna C, Safeukui I, Bonnet S: Plasmodium falciparum transmission blocking immunity in three areas with perennial or seasonal endemicity and different levels of transmission. Am J Trop Med Hyg 2005, 73:1090-1095.

25. Charlwood JD: Biological variation in Anopheles darlingi Root. Mem Inst Oswaldo Cruz 1996, 91:391-398.

26. Schneider CA, Rasband WS, Eliceiri KW: NIH Image to ImageJ: 25 years of image analysis. Nat Methods 2012, 9:671-675.

27. Briegel $\mathrm{H}$ : Determination of uric acid and hematin in a single sample of excreta from blood-fed insects. Experientia 1980, 36:1428.

28. Lehmann T, Dalton R, Kim EH, Dahl E, Diabate A, Dabire R, Dujardin JP: Genetic contribution to variation in larval development time, adult size, and longevity of starved adults of Anopheles gambiae. Infect Genet Evol 2006, 6:410-416.

29. Ameneshewa B, Service MW: The relationship between female body size and survival rate of the malaria vector Anopheles arabiensis in Ethiopia. Med Vet Entomol 1996, 10:170-172.

30. Yaro AS, Dao A, Adamou A, Crawford JE, Traore SF, Toure AM, Gwadz R, Lehmann T: Reproductive output of female Anopheles gambiae (Diptera: Culicidae): comparison of molecular forms. J Med Entomol 2006, 43:833-839.
31. Muira T, Takahashi RM: The fecundity of Aedes nigromacalus in the laboratory - effects of body weight and size of blood meal. Mosq News 1972, 32:417-421.

32. Albuquerque CM, Cavalcanti VM, Melo MA, Vercosa P, Regis LN, Hurd H: Bloodmeal microfilariae density and the uptake and establishment of Wuchereria bancrofti infections in Culex quinquefasciatus and Aedes aegypti. Mem Inst Oswaldo Cruz 1999, 94:591-596.

33. Briegel H: Fecundity, metabolism, and body size in Anopheles (Diptera: Culicidae), vectors of malaria. J Med Entomol 1990, 27:839-850.

34. Briegel $\mathrm{H}$ : Metabolic relationship between female body size, reserves, and fecundity of Aedes aegypti. J Insect Physiol 1990, 36:165-172.

35. Clements AN: The Biology of Mosquitoes, Volume l: Development,Nutrition and Reproduction. Oxforshire: Cabi Publishing; 2000.

36. Kelly DW: Why are some people bitten more than others? Trends Parasitol 2001, 17:578-581.

37. Lyimo IN, Ferguson HM: Ecological and evolutionary determinants of host species choice in mosquito vectors. Trends Parasitol 2009, 25:189-196.

doi:10.1186/1475-2875-12-2

Cite this article as: Sangare et al:: Studying fitness cost of Plasmodium falciparum infection in malaria vectors: validation of an appropriate negative control. Malaria Journal 2013 12:2.

\section{Submit your next manuscript to BioMed Central and take full advantage of:}

- Convenient online submission

- Thorough peer review

- No space constraints or color figure charges

- Immediate publication on acceptance

- Inclusion in PubMed, CAS, Scopus and Google Scholar

- Research which is freely available for redistribution 